Research Article

\section{Synthesis of $\mathrm{NaYF}_{4}: \mathrm{Yb}, \mathrm{Er} @ \mathrm{SiO}_{2} @ \mathrm{Ag}$ core-shell nanoparticles for plasmon- enhanced upconversion luminescence in bio-applications}

\section{Grigory Arzumanyan ${ }^{1,2 *}$, Dmitriy Linnik ${ }^{3 *}$, Kahramon Mamatkulov ${ }^{1}$, Maria Vorobyeva ${ }^{1}$, Anastasia Korsun ${ }^{4}$, Valentina Glasunova ${ }^{5}$ and Anka Jevremović 6}

\author{
'Laboratory of Neutron Physics, Joint Institute for Nuclear Research, 6 Joliot-Curie Str. Dubna \\ 141980, Russia \\ ${ }^{2}$ Dubna State University, 19 Universitetskaya str. Dubna, 141982, Russia \\ ${ }^{3}$ Donetsk National University, 17a Schorsa Str. Donetsk, Ukraine \\ ${ }^{4}$ Dotetsk National Medical University after M.Gorkiy, 16 Illica Ave., Donetsk, Ukraine \\ ${ }^{5}$ Donetsk Institute for Physics and Engineering named O.Galkin, 72 R. Luxemburg Str. Donetsk, \\ Ukraine \\ ${ }^{6}$ University of Belgrade, Faculty of Physical Chemistry, Studentski trg 12-16, 11158, Belgrade, \\ Serbia
}

\section{Abstract}

The present report highlights our results on synthesis of $\mathrm{NaYF}_{4}: \mathrm{Yb}_{1} \mathrm{Er}_{\mathrm{CSiO}} @ \mathrm{Ag}$ core-shell nanoparticles (CSNPs) for plasmon-enhanced upconversion luminescence (UCL). Hydrophilic surface UCL nanoparticles (UCLNPs) as cores were obtained by precipitation of Rare Earth Elements (REE) chlorides from water-alcohol solutions. The formation of a hydrophobic surface of a-NaYF $: \mathrm{Yb}_{4} \mathrm{Er} \mathrm{NPs}$ was achieved by thermolysis method at $280{ }^{\circ} \mathrm{C}$ and $\beta-\mathrm{NaYF}_{4}: \mathrm{Yb}, \mathrm{Er}$ by precipitation method in nonpolar medium at $320^{\circ} \mathrm{C}$. Silica shell was formed by the modified Stöber method on the surfaces of UCLNPs with different polarity and phase composition. A mixture of hexane-cyclohexane-isopropyl alcohol was used as a medium for the formation of mononuclear CSNPs on hydrophobic surfaces of cores with different thicknesses of the silica shell: $5 \mathrm{~nm}$ and $14 \mathrm{~nm}$. Formation of a predetermined thickness of silica shell was carried out by introducing a precise quantity of TEOS taking into account the size of core NPs with molar ratio TEOS: $\mathrm{H}_{2} \mathrm{O}$ equal to 1:6. The morphology and phase composition of cores and CSNPS were examined by transmission electron microscopy and selected area electron diffraction, respectively. The insertion of $\mathrm{Ag} \mathrm{NPs}$ into the structure of $\mathrm{NaYF}_{4}: \mathrm{Yb}, \mathrm{Er} @ \mathrm{SiO}_{2}$ was carried out in parallel at the stage of shell formation, which made this synthesis a one-step process. The control of the size of Ag NPs was implemented through the use of a colloidal solution of NPs of the cluster structure by changing the polarity of the medium. The highest intensity enhancement of 85 -fold with $5 \mathrm{~nm}$ and 29 -fold with $14 \mathrm{~nm}$ shell thickness was recorded, respectively. For the first time, tests on bioimaging of neutrophil cells by those CSNPs are demonstrated.

\section{More Information}

*Address for Correspondence: Grigory Arzumanyan, Laboratory of Neutron Physics, Joint Institute for Nuclear Research, 6 JoliotCurie Str., Dubna 141980, Russia, Tel: +7 916925 7307; Email: arzuman@jinr.ru

Dmitriy Linnik, Donetsk National University, 17a Schorsa Str. Donetsk, Ukraine, Email: linnikus_911@mail.ru

Submitted: 27 September 2019

Approved: 11 October 2019

Published: 12 October 2019

How to cite this article: Arzumanyan G, Linnik D, Mamatkulov K, Vorobyeva M, Korsun A, et al. Synthesis of $\mathrm{NaYF}_{4}: \mathrm{Yb}, \mathrm{Er} @ \mathrm{SiO}_{2} @ A g$ coreshell nanoparticles for plasmon-enhanced upconversion luminescence in bio-applications. Ann Biomed Sci Eng. 2019; 3: 013-019.

DOI: 10.29328/journal.abse.1001006

Copyright: (c) 2019 Arzumanyan G, et al. This is an open access article distributed under the Creative Commons Attribution License, which permits unrestricted use, distribution, and reproduction in any medium, provided the original work is properly cited

Check for updates

\section{Introduction}

Development of high-techs claims production of stable, energy-conserving and eco-friendly equipment. One of such techniques in optics is based on the upconversion effect which involves the conversion of two or more lower-energy photons into one higher-energy photon. According to the literature, the upconversion process were systematically developed by N. Bloembergen and F. Auzel since 1959 [1-3].
The main difference between upconversion nanoparticles based on nanomaterials doped with lanthanide ions and other materials (e.g. quantum dots) is their visible-light emission under near infrared irradiation via nonlinear optical process [4-6]. To date the interest to the phenomenon of upconversion has received a new impulse due to the synthesis of modern matrix elements that contain various nanocomposites as well as core-shell $[7,8]$. 
Rare earth ions and particularly $\mathrm{Er}^{3+}$ ion is the most recognized and often used for UCL purposes. Nearly equidistant energy levels of $\mathrm{Er}^{3+}$ make it proper suited for UCL excitation and thus desirable as an activator in many different hosts [9]. Host materials must have low phonon energies to avoid efficiency loses through nonradiative transfer and should be chemical stable [10]. Rare earth fluorides are among the most promising and widely used host material meeting the requirements for efficient UCL phosphors [11]. Those advantages can lead to low probability of nonradiative decay and increased luminescence quantum yield.

Three common methods for synthesis of UCLNPs: thermal decomposition [12,13], hydrothermal synthesis [14] and co-precipitation in nonpolar medium [15]. Thermal decomposition can give well shaped particles with good size control including the relatively short period of reaction. It usually requires usage of surfactants with dissolving organic precursors in high-boiling organic solvents. Rare earth-doped $\mathrm{NaYF}_{4}$ nanocrystals (NCs) having low-energy phonon modes about $350 \mathrm{~cm}^{-1}$ are known to be a very efficient near-infrared to visible upconverters and can successfully play a role of luminescent cores in the formation of CSNPs [10]

CSNPs as a special class of biphasic nanostructured materials composed of the inner core and outer shell have been attracting increasing attention in recent years [16-18]. The synthesis of CSNPs has the ability to use a wide range of materials as a core and/or shell that can give a desired and unique properties and functions, such as physicochemical, biological, optical, etc. [19]. CSNPs are becoming more prominent in biological sciences an application based on the surface chemistry, which increases its affinity to bind with drugs, receptors, ligands etc $[20,21]$. Due to diverse chemical compositions, CSNPs have a number of advantages over simple nanoparticles such as less cytotoxicity, increase in dispersibility, bio- and cyto-compatibility, better conjugation with other bioactive molecules, increase of thermal and chemical stability $[22,23]$. This has led to the synthesis of novel CSNPs in synchronize with the biological system making them as one of the most attractive nanocomposites in the biomedical applications, including bioimaging, targeted drug delivery, photodynamic and photothermic therapy (PDT and PTT), sensors [24-30].

The main method for the synthesis of CSNPs is the solgel method developed by Stöber [31] and its modifications $[32,33]$, based on the hydrolysis of the organosilicon precursor with the subsequent formation of a silica shell on the surface of a functional material. In contrast to CSNPs obtained by thermolysis or deposition from a non-polar medium [34], formation of the silica shell on the polar surface of NPs can be carried without using a surfactant or complexing agent [35]

This report is devoted to the synthesis of $\mathrm{NaYF}_{4}: \mathrm{Yb}, \mathrm{Er} @$ $\mathrm{SiO}_{2} @ A g$ CSNPs with polar and non-polar surfaces of phosphor NPs by thermolysis and precipitation methods and their use in UCL studies.

\section{Experimental}

\section{Materials}

All chemicals were of analytical grade and used without further purification. Purity of the oxides of REE, $\mathrm{Y}_{2} \mathrm{O}_{3}, \mathrm{Yb}_{2} \mathrm{O}_{3}$ and $\mathrm{Er}_{2} \mathrm{O}_{3}$ was $99.99 \%$ and were purchased from LLC LANHIT, sodium fluoride (99.9\%), ammonium fluoride (98.16\%), glycerin (99\%), ethylene glycol (99\%), ethanol (99.9\%), isopropyl alcohol (99.7\%), acetone (99.98\%) and oleylamine (90\%) were purchased from LLC Himmed, hydrochloric acid (37\%), octadecen-1 (90\%), hexane for chromatography (99\%), tetraethoxyorthosilane (TEOS) (98\%), Igepal CO-520 (98\%) were purchased from Sigma Aldrich. Silver nitrate (99.9\%) was purchased from JSC LenReactive.

\section{Synthesis of core NPs: $\mathrm{NaYF}_{2}: \mathrm{Yb}, \mathrm{Er}$}

Phosphor NPs with a polar surface were obtained by precipitation of REE precursors by sodium and ammonium fluorides from aqueous solutions. To control the morphology and size of the particles, an alcoholic co-solvent was used which also served as a complexing agent. The aging time of the particles in the solution was 10 minutes which is known as typical for precipitation method [36] and additionally confirmed by the SEM images which we do not present in this article. Then the colloidal solution was centrifuged and washed with distilled water (Figure 1a,b). To obtain compacts NPs, acetone was added to the wet paste and dispersed after which the precipitate was centrifuged and dried at $60^{\circ} \mathrm{C}$. The synthesized nanoparticles had a fluorite type cubic structure $(\alpha$-phase) (Figure 1c).

Particles with non-polar surface were formed by various methods depending on the required size or morphology. Thermolysis method was used to obtain particles with a size less than $50 \mathrm{~nm}$. Methanol solutions of REE trifluoroacetate and sodium in a molar ratio of 1:1.5 were mixed with oleylamine and octadecene. The mentioned ratio was chosen with the aim to stabilize the process of phase formation of $\mathrm{NaYF}_{4}$ hexagonal structure - a well-known approach based on the phase diagram of NaF-YF ${ }_{3}$ [37]. Salt concentrations were $1 \mathrm{~mol} \mathrm{l}^{-1}$. Mixture was stirred, evacuated for 15 minutes at $150{ }^{\circ} \mathrm{C}$ and then heated at $280-340^{\circ} \mathrm{C}$. The variation in the
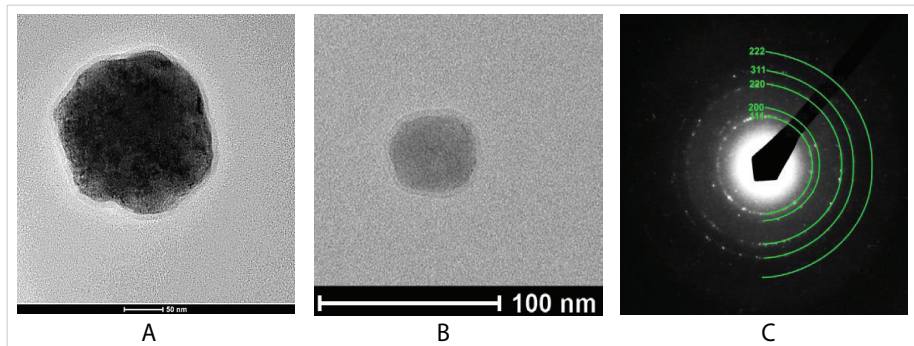

Figure 1: Shows TEM images of CSNPs with different core sizes and $5 \mathrm{~nm}$ shell thicknesses obtained by precipitation in aqueous-alcoholic. TEM images of $\mathrm{NaYF}_{4}: \mathrm{Yb}, \mathrm{Er}_{\mathrm{SSiO}} \mathrm{CSNPs}_{2}$ with various core sizes: A) $\left.200 \mathrm{~nm}, \mathrm{~B}\right) \sim 45 \mathrm{~nm}$ and shell thicknesses of $5 \mathrm{~nm}, \mathrm{C}$ ) selected area electron diffraction (SAED) pattern. 
ratio of oleylamine and octadecene as well as the heating rate and synthesis time allowed controlling the morphology of the nanoparticles. A pure cubic phase was obtained at a synthesis temperature of $280^{\circ} \mathrm{C}$, and a pure hexagonal phase at $(320-340){ }^{\circ} \mathrm{C}$. The formation of larger NPs of phosphors was achieved by using the method of deposition from nonaqueous solutions at $330{ }^{\circ} \mathrm{C}$. A mixture of oleylamine and octadecene was used as a solvent (10:15ml). Imparting hydrophobicity to NPs obtained by precipitation from wateralcohol solutions was achieved as follows: a powder of 500 mg NPs was dispersed in $15 \mathrm{ml}$ of a mixture of octadecene and oleylamine (9:6). The colloidal solution was heated under the low pressure to $150{ }^{\circ} \mathrm{C}$ with constant stirring to remove water adsorbed on the surface of the nanoparticles. Colloidal solution was heated to $280{ }^{\circ} \mathrm{C}$ for 30 minutes, cooled to the room temperature, centrifuged and washed with isopropyl and hexane. Glycerin was added to a colloidal solution of phosphor nanoparticles in water to increase viscosity. This approach allowed us to avoid the aggregation of nanoparticles and the formation of polynuclear structures.

\section{Formation of $\mathrm{SiO}_{2}$ shell}

Ammonia and non-ionic surfactant (Igepal CO 520) was added to the resulting colloidal solution. Ammonia was used to create a weak basic environment in order to controllably speed up the process of hydrolysis of TEOS. The use of surfactant in this case can be avoided provided that thickness of the shell should not exceed $10 \mathrm{~nm}$. Organosilicon precursor tetraethoxyorthosilane (TEOS) was added to the solution. The formation of the shell occurred during the day with vigorous stirring. To increase the stability of the colloidal solution, isopropyl alcohol was partially replaced by cyclohexanol. In a colloidal solution of $\mathrm{NaYF}_{4}: \mathrm{Yb}, \mathrm{Er}$ nanoparticles where a mixture of hexane-cyclohexane-cyclohexanol was used as the medium, an ammonia solution and surfactant Igepal CO-520 were added. Silver nanoparticles were obtained by reducing silver nitrate with oleylamine. The obtained nanoparticles were separated from oleylamine by centrifuging and washed with cyclohexane and hexane. Isopropyl alcohol was added to a colloidal solution of silver nanoparticles in hexane, which in turn led to an increase in particle size. Formation of the shell occurred within 24 hours.

\section{Characterization}

Morphology of nanoparticles was characterized by transmission electron microscopes (TEM) JEOL JEM-200A and Tecnai G2 80-200 X-Twin at an accelerated voltage 200 $\mathrm{kV}$. By electron diffraction patterns on TEM instruments, the phase composition of the obtained NPs was determined.

UCL studies were carried out using a confocal microspectroscopy set-up "Confotec" (SOL Instruments) which comprises laser spectrometer coupled to the NIKON TE2000-E inverted microscope. A continuously tunable between 690-990 nm wavelengths laser source (EKSPLA PT257) was exploit for the excitation of the UCL processes at $976 \mathrm{~nm}$. The laser beam was focused into the sample by $40 \times(\mathrm{NA}=0.6)$ microscope objective lens. The samples were located at the motorized sample position adjustment stage (Prior Scientific, H117TE). The UCL spectra were analyzed with the MS-520 monochromator-spectrograph coupled to a Peltier-cooled CCD camera (ProScan HS101H). To block the laser radiation, we used FF01 890/SP-25 edge short-pass emission filter (Semrock). All measurements were performed at room temperature.

\section{Results and Discussion}

Most of the studies on the synthesis of luminescent NPs of the core-shell structure are devoted to materials with a non-polar surface of the phosphor NPs. At the same time, a small number of works are devoted to the formation of these structures for systems with a polar surface of the NPs by the Stöber method [38]. It was revealed that the formation of silica shell on the surface of polar NPs is not a complicated task even in aqueous solutions [39].

In order to avoid NPs aggregation and acid hydrolysis of the yttrium tetrafluorides matrix, the use of a weakly basic medium was necessary.

Core NPs obtained by precipitation from a water-glycerin solution of REE chlorides and the other ones from a waterethanol solution are characterized by an average size of 190$220 \mathrm{~nm}$ and 40-55 $\mathrm{nm}$, respectively. By varying the ratio of the number of core NPs and the volume of TEOS the thickness of the forming shell was varied.

It is worth nothing that the formation of core-shell structures on the basis of particles with a non-polar surface is more difficult task, since not only an increase in the dispersibility of particles in a polar environment is required, but also the size of particles plays an important role in the formation of these structures. Figure 2a shows TEM images of $\mathrm{NaYF}_{4}: \mathrm{Yb}$,Er core NPs obtained by the precipitation of rare earth chlorides with a methanol solution of sodium hydroxide and ammonium fluoride in a mixture of octadecene-oleylamine. However, this approach does not lead to the formation of mononuclear core-shell structures for large NPs (Figure $2 \mathrm{~b}$ ) even with the insertion of a large amount of surfactant. This is mainly due to the aggregation of $\mathrm{NaYF}_{4}: \mathrm{Yb}$,Er NPs in a colloidal solution with the introduction of a polar medium ammonia solution. Increase in dispersibility of the NPs can be achieved by partial replacement of hexane with cyclohexane and reducing NPs size.

Phosphor NPs with an average size of $30 \mathrm{~nm}$ were synthesized by thermolysis of REE and sodium trifluoroacetates in a mixture of octadecene and oleylamine. As in the case of the precipitation from non-polar medium, these NPs are characterized by a hydrophobic surface. 
In the synthesis of core-shell structures with $90 \%$ replacement of hexane by cyclohexane, bimodal structures are formed - polynuclear (Figure 3a) and mononuclear (Figure 3b) CSNPs. The mechanism of aggregation of NPs in a colloidal solution is the same as in the previous case, since the polarity of cyclohexane remains low. The surfactant content was $2 \mathrm{vol} \%$. An increase in the content of surfactants up to 25 vol\% (Figure 4a), particle size or their content in a colloidal solution (Figure $4 \mathrm{~b}$ ) was not actually lead to a decrease in the yield of the fraction of polynuclear structures. However, it should be noted that with an increase in the surfactant content and a decrease in the concentration of NPs in the colloidal solution, the dispersibility of the nanoparticles increased, as can be seen from the micrographs. Aggregates of NPs associated with an increase in the polarity of the medium are no longer observed. Phosphor NPs were completely covered by a silica shell and were not interact among themselves.

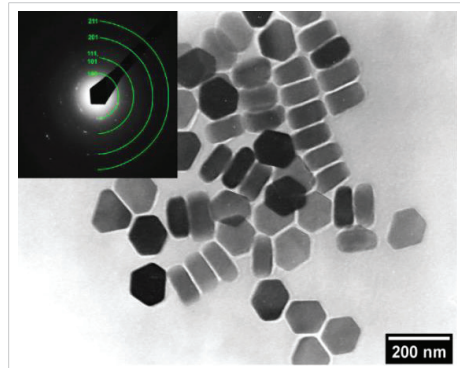

A

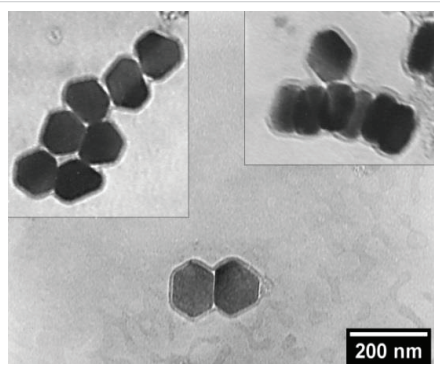

B
Figure 2: TEM images of A) NaYF $: \mathrm{Yb}_{4}$ Er NPs, obtained by the precipitation in a non-polar medium and B) the resulting polynuclear structures $\mathrm{NaYF}_{4}: \mathrm{Yb}_{\text {, }} \mathrm{Er}_{\mathrm{SSiO}}$

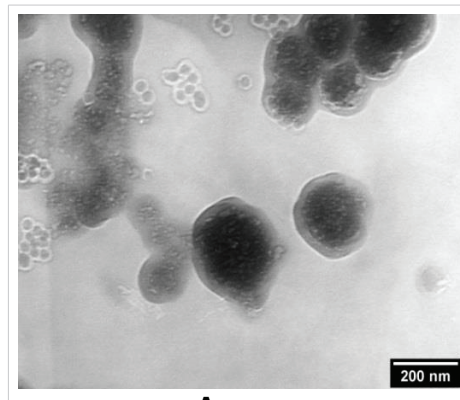

A

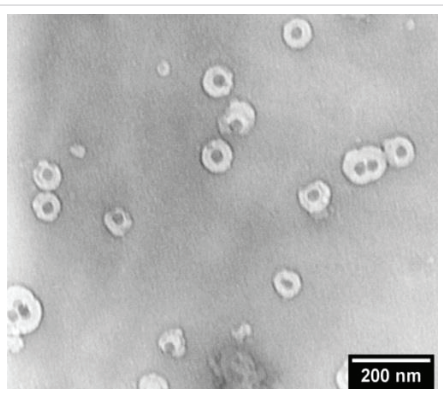

B
Figure 3: TEM images of A) polynuclear and (B)mononuclear CSNPs obtained by partial replacement of hexane with cyclohexane.

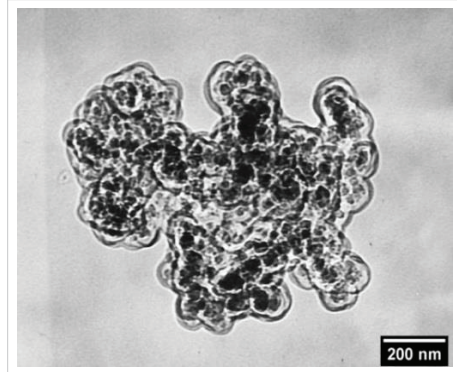

A

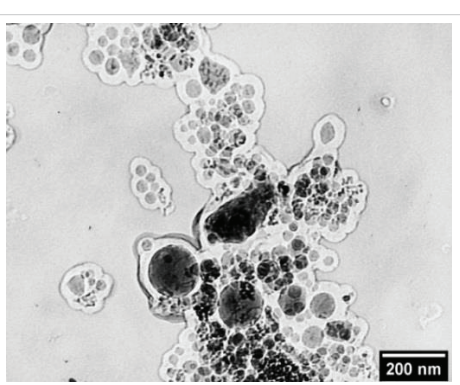

B
Figure 4: TEM images of polynuclear structures $\mathrm{NaYF}_{4}: \mathrm{Yb}_{1} \mathrm{Er}_{\mathrm{SSiO}}$ obtained with (A) an increase in the Igepal CO-520 surfactant content and (B) a decrease in the concentration of NPs in a colloidal solution
The conservation of the polynuclear structure can be explained as follows. During the hydrolysis of TEOS a shell was formed on the surface of the nanoparticles. Polar surface of the nanoparticles was containing a lot of polar hydroxyl groups. An increase in the polarity of the particles leads to their aggregation since the polarity of the hexane-cyclohexane medium was low. During the subsequent hydrolysis of TEOS, the shell was growing on aggregates of $\mathrm{NaYF}_{4}: \mathrm{Yb}, \mathrm{Er} @ \mathrm{SiO}_{2} \mathrm{NPs}$.

Thus, it was concluded that it is necessary to use a solvent with a high polarity, but in which aggregation of phosphor NPs with a hydrophobic surface would not occur. A triple mixture based on hexane, cyclohexane and isopropyl alcohol was chosen as such a solvent. The surfactant content in this case was less than $25 \%$ vol. Thus, it was possible to form mononuclear core-shell structures $\mathrm{NaYF}_{4}: \mathrm{Yb}, \mathrm{Er} @ \mathrm{SiO}_{2}$. Changing the TEOS content was led to allows one to vary the shell thickness variation.

An increase in the content of TEOS leads to the need to increase the water content in the colloidal solution, which previously led to the formation of aggregates of NPs and the formation of a polynuclear structure. (Figure $5 \mathrm{a}, \mathrm{b}$ ) shows micrographs of mononuclear core-shell structures obtained in a colloidal solution based on the solvent hexane-cyclohexaneisopropyl alcohol (20:40:40 vol\%) with different core sizes about 120 and $100 \mathrm{~nm}$ and shell thickness $14 \mathrm{~nm}$ and $28 \mathrm{~nm}$, respectively.

To strengthen the UCL emission of the synthesized CSNPs, we applied plasmonic enhancement method by the use of modified $\mathrm{SiO}_{2}$ shell decorated with 8-12 nm silver nanoparticles. Usually, CSNPs were modified by additional condensation on the shell surface by organic molecules with terminal thiol or amine groups, which in turn could be strongly bonded to metal NPs [40]. This approach is complex and is characterized by several disadvantages such as multi-stage synthesis, low yield of the target product and reduced functionality of the shell surface for further chemical modifications.

Given the previously mentioned shortcomings we have proposed the direct embedding of AgNPs into the $\mathrm{SiO}_{2}$ shell in parallel to its formation which eliminates the multi-stage synthesis. Also, it should be noted, that in the present work we didn't put a goal to study the AgNPs size-effect on the

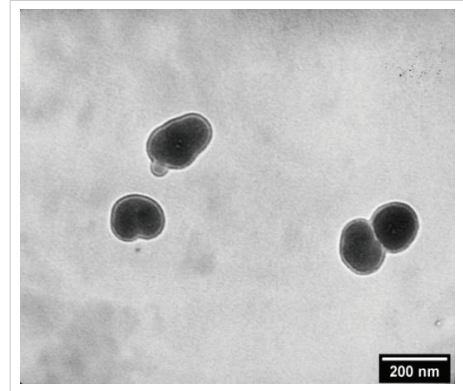

A

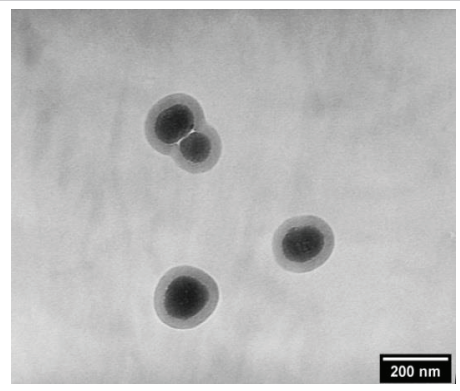

B
Figure 5: TEM images of mononuclear $\mathrm{NaYF}_{4}: \mathrm{Yb}_{\text {, }} \mathrm{Er} @ \mathrm{SiO}_{2}$ with different size of phosphor NPs and shell thickness: A) $120 \mathrm{~nm} @ 14 \mathrm{~nm}$, B) $100 \mathrm{~nm} @ 28 \mathrm{~nm}$. 
UCL intensity leaving this for the further investigations. The use of AgNPs in a cluster structure made it possible to vary their size due to a change in the polarity of the medium. A colloidal solution of AgNPs was added to a mixture of hexanecyclohexane-isopropyl alcohol followed by the formation of a $\mathrm{SiO}_{2}$ shell, the size of AgNPs increased from $3 \mathrm{~nm}$ to 10-12 nm.

As a result of AgNPs embedding to our CSNPs the UCL yield was enhanced for 85 and 29 times for structures with a shell thickness of $5 \mathrm{~nm}$ and $14 \mathrm{~nm}$, respectively (Figure 6a,b). Though the $5 \mathrm{~nm}$ shell demonstrates almost 3 times stronger UCL intensity in the red band of the emission in comparison with $14 \mathrm{~nm}$ shell, on the other hand, given the fact that the shell of silicon dioxide was acting as a diffusion barrier between the surface of the phosphor NPs and the polar environment (especially water) a stronger protection of the phosphor core from degradation could be achieved in structures with a larger thickness of the shell.

We implemented tests on UCL bioimaging of neutrophil cells. Neutrophil cells were immersed in saline with $\mathrm{NaYF}_{4}: \mathrm{Yb}, \mathrm{Er} @ \mathrm{SiO}_{2} @ \mathrm{Ag} \mathrm{CSNPs}$ for 30 minutes to capture some quantity of phosphor NPs. Next, neutrophils were deposited on a microscope coverslip and fixed with an alcohol solution twice washed with distilled water and drying at room temperature. Figure 7 shows UCL image of neutrophil cell taken at the red band of the luminescence spectrum.

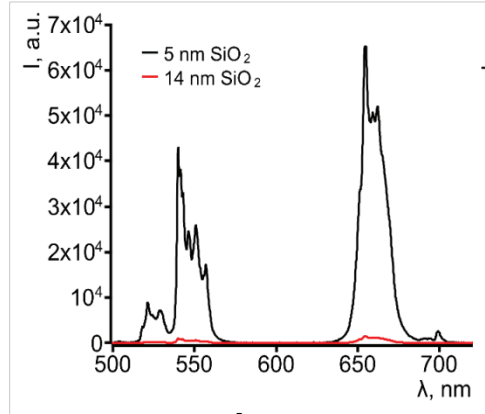

A

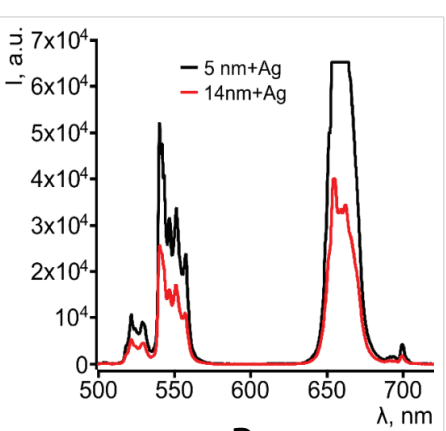

B
Figure 6: UCL spectra of CSNP A) $\mathrm{NaYF}_{4}: \mathrm{Yb}, \mathrm{Er}_{\mathrm{OSSiO}}$ and B) NaYF$: \mathrm{Yb}, \mathrm{Er}_{4} @ \mathrm{SiO}_{2} @ A g$ with two different thicknesses of $\mathrm{SiO}_{2}$ shell.

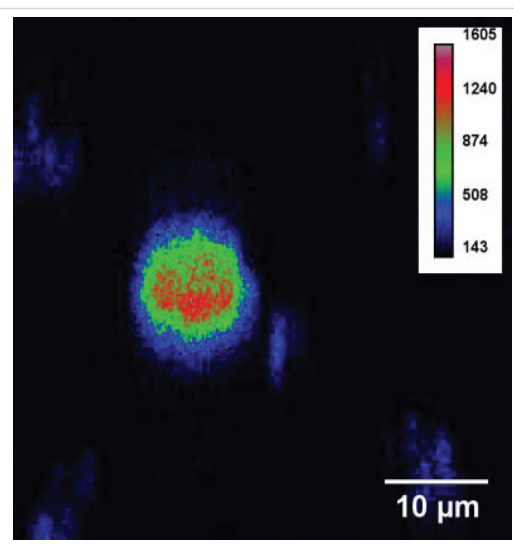

Figure 7: UCL image of neutrophil cells.

\section{Conclusion}

Core-shell nanoparticles $\mathrm{NaYF}_{4}: \mathrm{Yb}, \mathrm{Er} @ \mathrm{SiO}_{2}$ with cubic and hexogonal core structures were successfully synthesized for plasmon-enhanced up-conversion luminescence and further were tested in bioimaging by modified Stöber method, which was very versatile for the synthesis of these type core-shell nanocomposites. Core NPs with a particle size of 50 to 200 $\mathrm{nm}$ and a polar surface were obtained by precipitation from a water-alcohol medium. NPs with a non-polar surface up to 50 $\mathrm{nm}$ in size were predominantly obtained by thermolysis, and larger particles were precipitated from a non-polar medium. Formation of a $\mathrm{SiO}_{2}$ shell on the surfaces protected cores from degradation in a polar environment, especially in protolytic solvents. The Stöber method was very versatile for the synthesis of these type core-shell nanocomposites. The use of different polarities ternary mixtures of solvents of different polarities made it possible to eliminate the formation of polynuclear structures regardless of the shell thickness and the size of the luminescent core NPs themselves. For the cores synthesized by the precipitation from a non-polar medium and by thermolysis, the optimal medium for the formation of a $\mathrm{SiO}_{2}$ shell was a mixture of hexane-cyclohexane-isopropyl alcohol.

CSNPs $\mathrm{NaYF}_{4}: \mathrm{Yb}, \mathrm{Er} @ \mathrm{SiO}_{2} @ \mathrm{Ag}$ with $\mathrm{SiO}_{2}$ shell thicknesses of 5 and $14 \mathrm{~nm}$ a UCL enhancement of 85-fold and 29-fold was observed, respectively. The first bioimaging test of synthesized CSNPs as intracellular bioprobes, showed the possibility of obtaining contrast bioimages using neutrophil cells as an example.

\section{References}

1. Bloembergen N. Solid state infrared quantum counters. Phys Rev Lett. 1959; 2: 84 .

2. Auzel F. Quantum counter by transfer of energy between two rare earth ions in mixture of tungstate and glass. C R Acad Sci (Paris). 1966; 262: 1016.

3. Auzel F. Quantum counter by energy transfer from $\mathrm{Yb} 3+$ to $\mathrm{Tm} 3+$ in a mixed tungstate and a germanate glass. C R Acad Sci (Paris). 1966; 263: 819-821.

4. Vennerberg D, Lin Z. Upconversion nanocrystals: synthesis, properties, assembly and applications. J Sci Adv Mater. 2011; 3: 26-40.

5. Cheng L, Wang C, Liu Z. Upconversion nanoparticles and their composite nanostructures for biomedical imaging and cancer therapy. Nanoscale. 2013; 5, 23-37.

PubMed: https://www.ncbi.nlm.nih.gov/pubmed/23135546

6. Liu Q, Feng W, Sun Y, Li R. Upconversion Luminescent Materials: Advances and Applications. Chem Rev. 2015; 115: 395-465. PubMed: https://www.ncbi.nlm.nih.gov/pubmed/25492128

7. Chen J, Zhao JX. Upconversion nanomaterials: synthesis, mechanism, and applications in sensing. IEEE Sens J. 2012; 12: 2414-2435.

8. Chen X, Peng D, Ju Q, Wang F. Photon upconversion in core-shell nanoparticles. Chem Soc Rev. 2015; 44: 1318-1330.

PubMed: https://www.ncbi.nlm.nih.gov/pubmed/25058157 
9. Meijerink A, Wegh RT. VUV Spectroscopy of Lanthanides: Extending the Horizon. Materials Science Forum. 1999; 315-317: 11-26.

10. Zhang F. Photon upconversion nanomaterials. New York. Springer. 2014.

11. Wang ZL, Chan HLW, Li HL, Hao JH. Highly efficient low-voltage cathodoluminescence of $\mathrm{LaF3}: \mathrm{Ln} 3+(\mathrm{Ln}=\mathrm{Eu} 3+, \mathrm{Ce} 3+, \mathrm{Tb} 3+)$ spherical particles. Applied Physics Letters. 2008; 93: 141106.

12. Sun X, Zhang YW, Du YP, Yan ZG, Si R, et al. From Trifluoroacetate Complex Precursors to Monodisperse Rare-Earth Fluoride and Oxyfluoride Nanocrystals with Diverse Shapes through Controlled Fluorination in Solution Phase. Chemistry - A European J. 2007; 13: 2320-2332.

PubMed: https://www.ncbi.nlm.nih.gov/pubmed/17163562

13. Li C, Lin J. Rare earth fluoride nano-/microcrystals: synthesis, surface modification and application. Journal of Materials Chemistry. 2010; 20: 6831.

14. Li C, Yang J, Quan Z, Yang P, Kong V, Lin J, et al. Different Microstructures of $\beta$-NaYF4 Fabricated by Hydrothermal Process: Effects of $\mathrm{pH}$ Values and Fluoride Sources. Chemistry of Materials. 2007; 19: 4933-4942.

15. Shi $F$, Zhao Y. Sub-10 nm and monodisperse $\beta$-NaYF4:Yb,Tm,Gd nanocrystals with intense ultraviolet upconversion luminescence. $J$ Mater Chem C. 2014; 2: 2198-2203.

16. López-Lorente Al, Simonet BM, Valcárcel M. Analytical Potential of Hybrid Nanoparticles. Anal Bioanal Chem. 2011; 399: 43-54. PubMed: https://www.ncbi.nlm.nih.gov/pubmed/20821000

17. Ghosh Chaudhuri R, Paria S. Core/Shell Nanoparticles: Classes, Properties, Synthesis Mechanisms, Characterization, and Applications. Chem Rev. 2012; 112: 2373-2433.

PubMed: https://www.ncbi.nlm.nih.gov/pubmed/22204603

18. Li JF, Zhang YZ, Ding SY, Rajapandiyan Panneerselvam, Zhong-Qun Tian, et al. Core-shell Nanoparticle Enhanced Raman Spectroscopy. Chem Rev. 2017; 117: 5002-5069.

PubMed: https://www.ncbi.nlm.nih.gov/pubmed/28271881

19. Khatami M, Alijani HQ, Nejad MS, Varma RS. Core/shell Nanoparticles: Greener Synthesis Using Natural Plant Products. Appl Sci. 2018; 8: 2-17.

20. Sahoo SK, Labhasetwar V. Nanotech approaches to drug delivery and imaging. Drug Discov Today. 2003; 8: 1112-20.

PubMed: https://www.ncbi.nlm.nih.gov/pubmed/14678737

21. Gilmore JL, Yi X, Quan L, Kabanov AV. Novel nanomaterials for clinical neuroscience. J Neuro Immune Pharmacol. 2008; 3: 83-94.

PubMed: https://www.ncbi.nlm.nih.gov/pmc/articles/PMC2566785/

22. Chatterjee K, Sarkar S, Jagajjanani Rao K, Paria S. Core/shell nanoparticles in biomedical applications. Advances in colloidal and Interface science. 2014; 209: 8-39.

PubMed: https://www.ncbi.nlm.nih.gov/pubmed/24491963

23. Liu W, Zhu Z, Deng K, Li Z, Zhou Y, et al. Gold Nanorod@Chiral Mesoporous Silica Core-shell Nanoparticles with Unique Optical Properties. JACS. 2013; 135: 9659-9664.

PubMed: https://www.ncbi.nlm.nih.gov/pubmed/23742128

24. Xiong LQ, Yang TS, Yang $Y, X u C$, Li F, et al. Long-term in vivo biodistribution imaging and toxicity of polyacrylic acid-coated upconversion nanophosphors. Biomaterials. 2010; 31: 7078-7085. PubMed: https://www.ncbi.nlm.nih.gov/pubmed/20619791

25. Zhang P, Steelant W, Kumar M, Scholfield M. Versatile photosensitizers for photodynamic therapy at infrared excitation. J Am Chem Soc. 2007; 129: 4526-4527.

PubMed: https://www.ncbi.nlm.nih.gov/pubmed/17385866
26. Qian HS, Guo HC, Ho PC, Mahendran R, Zhang Y, et al. MesoporousSilica-Coated Up-Conversion Fluorescent Nanoparticles for Photodynamic Therapy. Small. 2009; 5: 2285-2290.

PubMed: https://www.ncbi.nlm.nih.gov/pubmed/19598161

27. Chen GY, Shen J, Ohulchanskyy TY, Patel NJ, Kutikov A, et al. (a-NaYbF$: \mathrm{Tm}+$ ) $/ \mathrm{CaF}_{2}$ Core/Shell Nanoparticles with Efficient NearInfrared to Near-Infrared Upconversion for High-Contrast Deep Tissue Bioimaging, ACS Nano. 2012. 6; 8280.

PubMed: https://www.ncbi.nlm.nih.gov/pubmed/22928629

28. Liu Q, Sun $Y$, Yang T, Feng W, Li C, et al. Sub-10 nm Hexagonal Lanthanide-Doped $\mathrm{NaLuF}_{4}$ Upconversion Nanocrystals for Sensitive Bioimaging in vivo. J Am Chem Soc. 2011; 133: 17122.

PubMed: https://www.ncbi.nlm.nih.gov/pubmed/21957992

29. Shan G, Weissleder R, Hilderbrand SA. Upconverting Organic Dye Doped Core-Shell Nano-Composites for Dual-Modality NIR Imaging and Photo-Thermal Therapy. Theranostics. 2013; 3: 267-274. PubMed: https://www.ncbi.nlm.nih.gov/pmc/articles/PMC3630527/

30. Lucky SS, Idris NM, Li, Huang K, Soo KC, Zhang Y, et al. Titania coated Upconversion Nanoparticles for Near-Infrared Light Triggered Photodynamic Therapy, ACS Nano, 2015, 9(1), 191-205. PubMed: https://www.ncbi.nlm.nih.gov/pubmed/25564723

31. Stöber, Werner, Fink, Arthur, Bohn, Ernst, et al. Controlled growth of monodisperse silica spheres in the micron size range. $\mathrm{J}$ of Colloid Interface Science. 1968; 26: 62-69.

32. Van Blaaderen A, Van Geest J, Vrij A. Monodisperse colloidal silica spheres from tetraalkoxysilanes: Particle formation and growth mechanism. J of Colloid Interface Science. 1992; 154: 481-501.

33. Prouzet $E$, Boissière $C$. A review on the synthesis, structure and applications in separation processes of mesoporous MSU-X silica obtained with the two-step process. Comptes Rendus Chimie. 2005; 8: 579-596.

34. Liu S, Chen G, Ohulchanskyy TY, Swihart MT, Prasad PN. Facile Synthesis and Potential Bioimaging Applications of Hybrid Upconverting and Plasmonic NaGdF4: Yb3+, Er3+/Silica/Gold Nanoparticles. Theranostics. 2013; 3: 275-280.

PubMed: https://www.ncbi.nlm.nih.gov/pubmed/23606914

35. Anees Ansari A, Parchur AK, Kumar B. Rai SB. Influence of Shell Formation on Morphological Structure, Optical and Emission Intensity on Aqueous Dispersible NaYF4:Ce/Tb Nanoparticles. J Fluoresc. 2016; 26: 1151-1159.

PubMed: https://www.ncbi.nlm.nih.gov/pubmed/27207570

36. Fedorov PP, Osiko VV, Kuznetsov SV, Uvarov OV, Mayakova MN, et al. Nucleation and growth of fluoride crystals by agglomeration of the nanoparticles. Journal of Crystal Growth. 2014; 401: 63-66.

37. Fedorov PP, Kuznetsov SV, Voronov VV, Yarotskaya IV, Arbenina VV Soft chemical Synthesis of NaYF4 Nanopowders. J Inorg Chemistry. 2008; 53; 1681-1685.

38. Zhao T, Nguyen NT, Xie Y, Sun X, Li Q et al. Inorganic Nanocrystals Functionalized Mesoporous Silica Nanoparticles: Fabrication and Enhanced Bio-applications. Front Chem. 2017; 5: 118.

39. Graf C, Dirk LJ, Vossen, Imhof A, van Blaaderen A. A General Method To Coat Colloidal Particles with Silica. Langmuir. 2003; 19: 6693-6700.

40. Qian LP, Zhou LH, Too HP, Chow GM. Gold decorated NaYF4:Yb,Er/ $\mathrm{NaYF}_{4} /$ silica (core/shell/shell) upconversion nanoparticles for photothermal destruction of $\mathrm{BE}(2)-\mathrm{C}$ neuroblastoma cells. J Nanoparticle Res. 